\title{
Self-Control and Problematic Internet Use in College Students: The Chain Mediating Effect of Rejection Sensitivity and Loneliness
}

\author{
Zeping Fan (1)', Ming Chen², Yaping $\operatorname{Lin}^{3}$ \\ 'College of Teachers Education, Ningbo University, Ningbo, Zhejiang, People's Republic of China; ${ }^{2}$ The Second Clinical Medical School, Anhui Medica \\ University, Hefei, Anhui, People's Republic of China; ${ }^{3}$ School of Nursing, Ningbo College of Health Sciences, Ningbo, Zhejiang, People's Republic of \\ China
}

Correspondence: Zeping Fan, College of Teacher Education, Ningbo University, 818 Fenghua Road, Jiangbei District, Ningbo City, Zhejiang Province, 315000, People's Republic of China, Tel +86|3685848408, Fax +86-574-87600046, Email fanzeping@nbu.edu.cn

Purpose: To explore the relationship between self-control, rejection sensitivity, loneliness, and problematic internet use in college students.

Patients and Methods: A total of 725 college students were investigated using Self-control Scale, Rejection Sensitivity Questionnaire, Loneliness Scale, and Internet Addiction Scale.

Results: (1) Correlation analysis showed that self-control was negatively related to rejection sensitivity, loneliness, and problematic internet use; rejection sensitivity was positively related to loneliness and problematic internet use; loneliness was positively related to problematic internet use. (2) Chain mediating effect analysis showed that self-control can not only affect problematic internet use in college students, but also through three indirect paths, as follows: the mediating role of rejection sensitivity and loneliness, the chain mediating roles of rejection sensitivity and loneliness, and the mediating effect size, accounting for $9.76 \%, 20.73 \%$, and $4.88 \%$ of the total effect, respectively.

Conclusion: Rejection sensitivity and loneliness played a chain mediating role in the relationship between self-control and problematic internet use in college students.

Keywords: self-control, rejection sensitivity, loneliness, problematic internet use, PIU, college students

\section{Introduction}

The Internet is not only a tool, but also a social environment. ${ }^{1}$ College students acquire information from this digital environment, where they socialize, live, and broadly understand their culture. The Internet has become an indispensable part of college students' life and learning. ${ }^{2}$ According to the 2021 Statistics Report on the Development of Internet in China, ${ }^{3}$ there were over 1 billion Internet users in the country. The Internet penetration rate was as high as $71.6 \%$, with a pattern of year-on-year expansion. College students account for a large proportion of Internet users. The Internet positively affects the social development of college students by enhancing self-confidence, exploration and unity, and exposure to new ideas; however, it also carries the risk of excessive pathological use of the Internet, called Problematic Internet Use (PIU). ${ }^{4}$

PIU refers to uncontrollable Internet use behavior that affects social functioning. ${ }^{5,6}$ It includes the following characteristics: excessive use, often losing sense of time; withdrawal or experiencing a bad mood when the computer is not accessible; tolerance, or needing more time and equipment to meet Internet needs; and adverse consequences, such as lying, reduced work efficiency, and isolation. ${ }^{7}$ Studies have shown that adolescents with excessive Internet use (PIU) may be at greater risk of impaired social and emotional functioning. ${ }^{8}$ The characteristics of the Internet—offering anonymity, convenience, and escape, making communication more private, and shopping and gaming more convenientare important factors that influence the likelihood of PIU. ${ }^{9}$ The diagnosis of PIU is controversial. Indeed, the current 
model equates PIU (pathological use behavior) with other substance addiction, ignoring the characteristics of the Internet as a universal and necessary tool, which also has limitations. ${ }^{10}$ Several studies have shown that PIU is associated with a range of physical and mental problems in college students, such as emotional problems, maladjustment, impaired social ability, and memory loss. ${ }^{11-16}$ Therefore, studying the formation mechanism of PIU and preventing college students from falling into pathological Internet use is vital. ${ }^{17}$

Davis (2001) believed that PIU is a behavioral problem defined by uncontrolled Internet use, which has been recognized within academic circles and is widely adopted. ${ }^{18}$ Further, exploring the formation mechanism of PIU from the perspective of self-control is widely favored by researchers. Self-control usually refers to the process of adjusting cognitive, emotional, and behavioral responses to achieve personal goals. ${ }^{19}$ Indeed in previous studies pointed out that failure to control impulses can lead to addiction problems. ${ }^{20} \mathrm{~A}$ number of recent studies has shown that higher self-control is associated with lower rates of Internet addiction. ${ }^{21-23}$ However, most of these studies regarded selfcontrol as a mediating variable and explored the correlation between self-esteem, depression, loneliness, and PIU through the role of self-control. Few scholars explored the direct correlation between self-control and PIU; further, self-control itself is a multi-system ability structure. The conflict between self-control and impulsive behavior is closely related to factors, such as the individual's personality. ${ }^{24}$ Self-control is a complex behavior pattern of biological consumption. The success of self-control is influenced by self-monitoring, ability, and other factors. ${ }^{20,25}$ The theory of self-control power model considers self-control a complex behavior pattern of biological consumption. ${ }^{26}$ It states that self-control is a process that consumes energy, and is limited and takes time to recover after consumption; its energy acts on all self-control modes (including cognition, emotion, and behavior); ${ }^{27}$ When the control power is exhausted, negative personality traits, such as social avoidance and anxiety, become prominent. ${ }^{28}$ These traits are strongly correlated with PIU. ${ }^{29,30}$ Therefore, we assume that self-control is not only directly related to PIU but is also related to it via certain trait variables. This study considers self-control as an independent variable and sets relevant mediating variables to analyze the path of self-control influencing PIU.

Rejection sensitivity is an individual's anxious anticipation and tendency to overreact to rejection signals from others. ${ }^{31}$ It is an important factor in the generation of depression, loneliness, and other emotions. ${ }^{32,33}$ Additionally, higher rejection sensitivity has been shown to be associated with higher social anxiety. ${ }^{34}$ Studies have indicated that social anxiety is closely related to PIU. ${ }^{35}$ Therefore, the latter has a greater correlation with rejection sensitivity. ${ }^{36}$ Another study noted that individuals with high rejection sensitivity could not experience high intimacy satisfaction because of their negative expected attitude toward rejection information. They tend to compensate for their lack of relationship needs through the Internet, thereby exhibiting stronger PIU behavior. ${ }^{37,38}$ The compensatory Internet use model theory notes that Internet addicts mainly use the Internet to alleviate negative emotions and escape from reality. Negative situations or lack of social activities in real life strengthen the motivation for using the Internet. Its use can alleviate negative emotions and satisfy the stimulation of individual communication. However, it can also encourage the development of PIU. ${ }^{39}$ According to this theory, the use of network can only compensate for the adverse consequences (eg, social anxiety) caused by rejection sensitivity. Knowles et al verified that the presentation of social media icons can effectively compensate for the basic psychological deficit caused by rejection sensitivity. ${ }^{40}$ Thus, individuals with high (vs lower) levels of rejection sensitivity may be more prone to PIU. According to the dual system (hot/cold) model theory of self-control, the result of self-control behavior is produced by the interaction of the impulsive, or hot, system and the reflexive, or cold, system. ${ }^{24}$ The hot system deals mainly with emotions (simple and fast) and produces impulses. The cold system deals primarily with cognition (complex, rational) and inhibits impulses. ${ }^{41}$ In interpersonal interactions, rejection sensitivity is an individual's transition response to the emotion of interpersonal interaction (hot system), and cognitive strategies of interpersonal interaction can effectively buffer this stimulus response (cold system). When there is no connection between hot and cold systems (ie, low self-control), the "rejected" node in the hot system results in the most impulsive and automatic responses (eg, hostility, which can be interpreted as higher rejection sensitivity). ${ }^{42}$ However, individuals with high (vs lower) self-control are more likely to use cognitive assessment strategies (cold strategies) to include non-rejection-related information, thus showing lower rejection sensitivity. ${ }^{43}$ Experimental studies have demonstrated that self-control is a protective factor against rejection sensitivity and can effectively reduce its 
negative effects. ${ }^{44}$ In conclusion, we can infer that self-control can be associated with PIU through the mediating role of rejection sensitivity. Therefore, we hypothesize that rejection sensitivity is a mediator between self-control and PIU.

Loneliness is a type of negative emotional experience caused by an interpersonal relationship gap and the resulting emotions, such as sadness, emptiness, and longing. ${ }^{45,46}$ According to Caplan, lonely individuals have a low selfevaluation of social skills and prefer online social interaction. This preference sets the stage for problematic web use. ${ }^{47}$ According to the Social Skill Account Model, ${ }^{48}$ lonely individuals prefer online interaction (as opposed to faceto-face interaction) due to their lack of social skills; indeed, they use online interaction as a "Prozac" for social interaction, ${ }^{30}$ which leads them to use the Internet compulsively, thus resulting in adverse outcomes (PIU). ${ }^{48}$ David et al believe that online social interaction is a "socially liberating" experience for such lonely individuals; consequently, they rely too much on the Internet as a tool to communicate with others, which makes it challenging to control their use of the Internet and leads to PIU. ${ }^{49}$ From the perspective of social needs theory, To make up for the lack of actual interpersonal relationship and to avoid dealing with the negative emotions caused by loneliness, individuals with high loneliness are more likely to overuse the Internet, which eventually leads to PIU. ${ }^{50}$ Empirical studies have also demonstrated the above conclusions. Several studies have confirmed that loneliness is highly correlated with the likelihood of PIU. ${ }^{51,52}$ From the perspective of social interaction, self-control can help strengthen behaviors of social bonds, such as cooperative behaviors, ${ }^{53}$ and reduce the possibility of aggressive reactions. ${ }^{54}$ Therefore, in interpersonal interaction, individuals with high self-control experience less social rejection and have a lower level of loneliness. By contrast, low self-control is often associated with negative personal outcomes and may lead to interpersonal rejection, which exacerbate feelings of loneliness. ${ }^{55}$ From the perspective of cognitive processing theory, loneliness is a negative emotion caused by individual perception of the gap between actual interpersonal relationship and expected interpersonal relationship; it is a product of subjective cognitive processing. ${ }^{56}$ As an advanced psychological activity, self-control can regulate or suppress unreasonable cognition, emotion, and behavior. Some studies have found that individuals with low self-control cannot overcome negative emotions and experience more loneliness. ${ }^{23}$ It can be speculated that self-control is associated with PIU through the mediating effect of loneliness. Consequently, we hypothesize that loneliness is also a mediator between self-control and PIU.

Additionally, rejection sensitivity is significantly correlated with loneliness. Several empirical studies have shown that individuals with high rejection sensitivity have a higher level of loneliness. ${ }^{33,34}$ According to the social cognitive model of rejection sensitivity, individuals' early experiences of rejection cause them to form an anxious expectation of rejection, which makes them alert to cues and behaviors that could signal rejection, thus leading them to adopt negative coping styles in interpersonal communication. This can seriously affect the establishment and maintenance of good personal relationships, causing them to become dissatisfied with the relationships they have formed and leading to feelings of loneliness. ${ }^{57}$ London also found that rejection sensitivity increases the experience of loneliness. ${ }^{34}$ Therefore, we hypothesize that self-control also influences PIU by rejection sensitivity and loneliness.

This study attempts to build a chain mediation model of rejection sensitivity and loneliness, further explores the psychological mechanism between self-control and PIU of college students, and provides certain theoretical reference for the prevention and reduction of PIU of college students. This study proposes the following hypothesis: self-control is not only directly related to PIU in college students but is also indirectly related through three paths:

1. Rejection sensitivity plays a mediating role in the relationship between self-control and PIU.

2. Loneliness is a mediator in the relationship between self-control and PIU.

3. Rejection sensitivity and loneliness together play a chain mediating role in the relationship between self-control and PIU.

\section{Methods}

\section{Participants}

Before the survey, a Monte Carlo power analysis for indirect effects was performed through an online application. ${ }^{58}$ The results show that a power of $0.80(p<0.05)$ is reached with 439 participants in a model with two serial mediators. We 
examined the relationship between self-control, rejection sensitivity, loneliness, and PIU in a larger sample to improve sensitivity. A total of 776 college students from Zhejiang province were randomly selected as survey participants. A total of 725 valid questionnaires were collected (effective response rate of 93.43\%). The sample included 286 men and 439 women, comprising 289 freshmen, 160 sophomores, 183 juniors, and 93 seniors. The mean age was 20.26 years $(\mathrm{SD}=$ 1.34 years). Of the participants, 382 were the only children of their parents, while 343 had siblings; 476 came from urban areas and 249 from rural areas; 424 students majored in science and engineering, while 301 majored in literature and history.

\section{Procedure}

After obtaining permission from the Zhejiang Provincial Department of Education, we commissioned teachers from five universities in Zhejiang province to assist us in conducting the questionnaire survey. We used a network format; we distributed questionnaires simultaneously and collected them within a limited time. Participants were informed of the purpose of the study before completing the questionnaire. They were informed that participation was voluntary, and that they could terminate their participation and withdraw from the survey at any time. Completing and submitting the questionnaire was regarded as their proxy consent to participate. All participants gave verbal informed consent.

\section{Measurement Tools}

\section{College Students' Self-Control Scale}

We adopted the college students' Self-control Scale, ${ }^{59}$ revised by Tan and Guo. ${ }^{38}$ This scale has 19 items, including 14 reverse-scored questions, with a 5-point score ( $1=$ "very inconsistent," and $5=$ "very consistent"). Higher scores indicate better self-control. The internal consistency coefficient of the scale was 0.84 .

\section{Rejection Sensitivity Questionnaire}

We adopted the Rejection Sensitivity Questionnaire, ${ }^{31}$ revised by Sun and $\mathrm{Wu} .{ }^{37}$ The questionnaire has 28 items, which includes 14 situations. Each situation has two questions (to assess the degree of concern regarding rejection $(1=$ "very unconcerned," 6 = "very concerned"), and the likelihood of rejection expected ( 1 = "very unlikely," 6 = "very likely"), which were scored on a six-point scale. In each situation, the "expected possibility of rejection" question was reverse scored (14 total). The sensitivity to rejection is calculated as follows: $\Sigma$ (degree of care for rejection by others $\times$ inverse score for the probability of expected rejection)/14. Higher scores indicate higher rejection sensitivity. The internal consistency coefficient of the questionnaire was 0.97 .

\section{Loneliness Scale}

We adopted the Loneliness Scale, ${ }^{60}$ revised by Wang. ${ }^{61}$ The scale has 20 items, including nine reverse-scored questions, with a 4-point score ( 1 = "never," 2 = "rarely," 3 = "sometimes," 4 = "always"). A higher score indicates a higher level of loneliness. The internal consistency coefficient of the scale was 0.95 .

\section{Internet Addiction Scale}

We used the Internet Addiction Scale, ${ }^{62}$ revised by Mei et al. ${ }^{63}$ The scale has 20 items, with a 5-point score $(1=$ "very inconsistent," $5=$ "very consistent"). A higher score indicates greater tendency of PIU. The internal consistency coefficient of the scale was 0.89 .

\section{Data Analysis}

\section{Common Method Deviation Test}

The data in this study were obtained using the questionnaire survey method, which may be affected by the common method bias, thereby resulting in the co-variation of human nature between the predictive variables and the outcome variables, and confusing or interfering with the actual research results and conclusions. ${ }^{64}$ Confirmatory factor analyses showed that the fitting degree of data and model was low $\left(\chi^{2} / \mathrm{df}=9.95\right.$, GFI $=0.18, \mathrm{AGFI}=0.14, \mathrm{IFI}=0.36, \mathrm{CFI}=0.36$, RMSEA $=0.11)$. Additionally, this study also adopted the single factor control method to test the common method deviation. ${ }^{65}$ The results showed no significant change in the fit of the model compared with the original model, after the 
addition of methodology factors $\left(\Delta \chi^{2} / \mathrm{df}=2.18 ; \Delta \mathrm{RMSEA}=0.002 ; \Delta \mathrm{CFI}=0.002 ; \Delta \mathrm{TLI}=0.009 ; \Delta \mathrm{SRMR}=0.009\right)$. Therefore, no serious common method deviation was found.

\section{Statistical methods}

SPSS 24.0 was used for descriptive statistics, reliability analysis, and correlation analysis. Pearson's correlations were conducted to describe the correlation coefficients between variables. Harman's single factor test and the single factor control method were used to test common method deviation. Then, the SPSS macro program process compiled by Hayes (2013) was adopted, Model 6 was selected according to templates, and 5000 samples were repeatedly extracted with a default confidence interval of $95 \%$. Using sex and age as control variables, we examined the chain mediating effects of rejection sensitivity and loneliness on self-control and PIU. If the $95 \%$ confidence interval of bootstrap does not contain 0 , it indicates that the mediating effects have reached significance.

\section{Results}

\section{Correlation Analysis of Self-Control, Rejection Sensitivity, Loneliness, and Problematic Internet Use}

Correlation analyses showed that self-control was negatively correlated with rejection sensitivity, loneliness, and PIU. Rejection sensitivity was positively correlated with loneliness and PIU. A significant positive correlation was also found between loneliness and PIU. Further, a significant correlation was observed between the variables, suitable for further mediating effect test. ${ }^{66}$ Additionally, gender and age were significantly correlated with other variables, except PIU. Thus, these demographic variables were considered control variables in subsequent analysis. The results are shown in Table 1.

\section{Chain Mediator Effect Test for Rejection Sensitivity and Loneliness}

Some researchers note that compared with sequential test and the Sobel method, the bootstrap method is more suitable for the test of chain multiple mediating effects. ${ }^{67-69}$ Therefore, the SPSS macro program process prepared by Hayes (2013) was adopted in this study. Based on the templates, we selected Model 6, repeatedly extracted 5000 samples, and the default confidence interval was $95 \%$. We used sex and age as control variables to examine the chain mediating effect of rejection sensitivity and loneliness on self-control and PIU. The negative effect of self-control on rejection sensitivity was significant (depression $=-0.41, P<0.001$ ); rejection sensitivity had a significant positive effect on loneliness (depression $=0.31, P<0.001$ ); self-control had a significant negative effect on loneliness (depression $=-0.61, P<$ 0.001 ); rejection sensitivity (depression $=0.20, P<0.001$ ) and loneliness (depression $=0.28, P<0.001$ ) were significantly positively correlated with PIU, whereas self-control was negatively correlated with PIU (depression $=$ $-0.53, P<0.001)$. The results are shown in Table 2 .

The results of the analysis of the mediating effect showed that rejection sensitivity and loneliness played a mediating role between self-control and PIU; the mediating effect was -0.29 , accounting for $35.37 \%$ of the total effect (of which the direct effect was -0.53 ); standard error is $0.04,95 \%$ confidence interval is $[-0.21,-0.06]$. Specifically, the mediating effect consists of three pathways, as follows: indirect pathway 1 (self-control $\rightarrow$ rejection sensitivity $\rightarrow$ PIU), standard error is $0.02,95 \%$ confidence interval is $[-0.12,-0.05]$; indirect pathway 2 (self-control $\rightarrow$ loneliness $\rightarrow$ PIU), standard error is

Table I Descriptive Statistics and Correlation Matrix of Each Variable $(n=725)$

\begin{tabular}{|c|c|c|c|c|c|c|c|c|}
\hline & $M$ & SD & $\mathbf{I}$ & 2 & 3 & 4 & 5 & 6 \\
\hline I Gender ${ }^{a}$ & - & - & 1.00 & & & & & \\
\hline 2 Age & 20.26 & 1.34 & $-0.10 * *$ & 1.00 & & & & \\
\hline 3 Self-control & 3.39 & 0.58 & $-0.13 * *$ & $0.11 * *$ & 1.00 & & & \\
\hline 4 Rejection Sensitivity & 10.01 & 2.81 & $0.10 * *$ & $-0.09 * *$ & $-0.42 * * *$ & 1.00 & & \\
\hline 5 Loneliness & 1.89 & 0.68 & $0.10 * *$ & $-0.08 * *$ & $-0.74 * * *$ & $0.56 * * *$ & 1.00 & \\
\hline 6 problematic internet use & 2.26 & 0.66 & -0.01 & -0.04 & $-0.64 * * *$ & $0.17^{* * *}$ & $0.56 * * *$ & 1.00 \\
\hline
\end{tabular}

Notes: Gender ${ }^{a}$ is the dummy variable, Male $=0$, Female $=1 . *_{p}<0.01, * * *_{p}<0.001$. 
Table 2 Chain Mediation Model Between Self-Control and Problematic Internet Use $(n=725)$

\begin{tabular}{|c|c|c|c|c|c|c|c|c|c|}
\hline \multirow[t]{2}{*}{ Variable } & \multicolumn{3}{|c|}{ Rejection Sensitivity } & \multicolumn{3}{|c|}{ Loneliness } & \multicolumn{3}{|c|}{ Problematic Internet Use } \\
\hline & $\boldsymbol{\beta}$ & SE & $\mathbf{t}$ & $\beta$ & SE & $\mathbf{t}$ & $\beta$ & SE & $\mathbf{t}$ \\
\hline Gender & 0.09 & 0.07 & 1.26 & -0.21 & 0.05 & -0.47 & -0.18 & 0.06 & $-3.20 *$ \\
\hline Age & -0.03 & 0.03 & -1.33 & 0.01 & 0.02 & 0.55 & 0.01 & 0.02 & 0.22 \\
\hline Self-control & -0.41 & 0.03 & $-11.94 * * *$ & -0.62 & 0.03 & $-23.98 * * *$ & -0.53 & 0.04 & $-13.04 * * *$ \\
\hline Rejection Sensitivity & & & & 0.31 & 0.03 & $|2.0|^{* * *}$ & 0.20 & 0.03 & $6.01 * * *$ \\
\hline Loneliness & & & & & & & 0.28 & 0.04 & $6.45^{* * *}$ \\
\hline $\mathrm{R}^{2}$ & \multicolumn{3}{|l|}{0.18} & \multicolumn{3}{|l|}{0.62} & \multicolumn{3}{|l|}{0.47} \\
\hline $\mathrm{F}$ & \multicolumn{3}{|c|}{$52.46 * * *$} & \multicolumn{3}{|c|}{$292.15 * * *$} & \multicolumn{3}{|c|}{$125.10 * * *$} \\
\hline
\end{tabular}

Notes: $*_{p}<0.05, * * *<<0.001$.

Table 3 Chain Mediated Effect Test of Rejection Sensitivity and Loneliness on Self-Control and PIU

\begin{tabular}{|l|l|l|l|l|l|}
\hline & Effect & BootSE & BootLLCI & BootULCI & $\begin{array}{l}\text { Relative } \\
\text { Mediation Effect }\end{array}$ \\
\hline $\begin{array}{l}\text { Indirect effect I } \\
\text { (Self-control } \rightarrow \text { Rejection } \\
\text { Sensitivity } \rightarrow \text { PIU) } \\
\text { Indirect effect 2 } \\
\text { (Self-control } \rightarrow \text { Loneliness } \rightarrow \text { PIU) } \\
\text { Indirect effect 3 } \\
\text { (Self-control } \rightarrow \text { Rejection } \\
\text { Sensitivity } \rightarrow \text { Loneliness } \rightarrow \text { PIU) } \\
\text { Total indirect effect }\end{array}$ & -0.08 & 0.02 & -0.12 & -0.05 & $9.76 \%$ \\
\hline
\end{tabular}

Notes: BootSE, BootLLCl, and BootULCI refer to the standard error of indirect effect estimated by percentile Bootstrap method of deviation correction and the lower and upper limits of the $95 \%$ confidence interval, respectively.

$0.04,95 \%$ confidence interval is $[-0.25,-0.11]$; and indirect pathway 3 (self-control $\rightarrow$ rejection sensitivity $\rightarrow$ loneliness $\rightarrow$ PIU), standard error is $0.01,95 \%$ confidence interval is $[-0.05,-0.06]$. The effect values of the three pathways were $-0.08,-0.17$, and -0.04 , accounting for $9.76 \%, 20.73 \%$, and $4.88 \%$ of the total effect, respectively. None of the bootstrap $95 \%$ confidence intervals of the three paths contained 0 , indicating that the three mediating effects reached a significant level. The results are shown in Table 3 and Figure 1.

\section{Discussion}

This study examined college students to explore the mechanism of the influence of self-control on PIU. Correlation analyses showed that self-control was negatively correlated with PIU, which is consistent with some recent studies. ${ }^{70,71}$

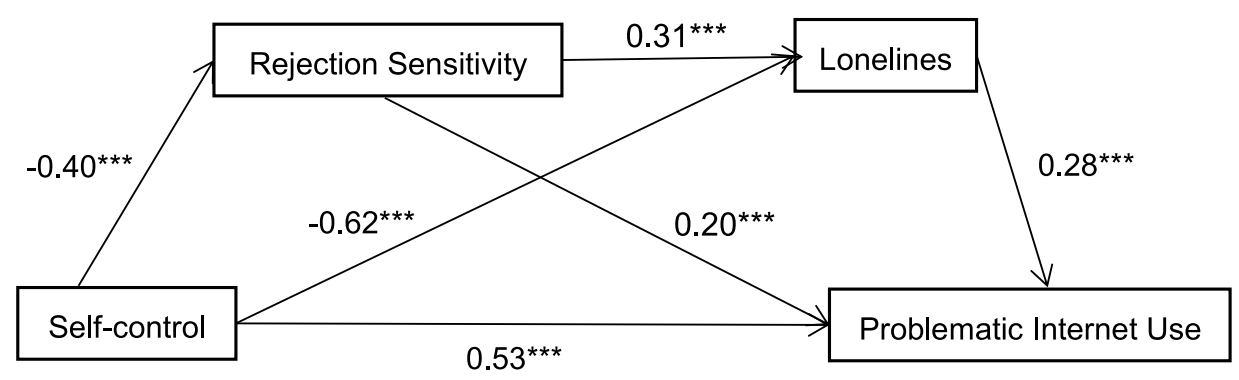

Figure I Schematic of chain mediation between rejection sensitivity and loneliness. ${ }^{* * *} p<0.00$ I. 
According to the general theory of crime by Gottfredson and Hirschi, ${ }^{72}$ lack of self-control is the main predictor of behavioral problems. PIU is the impulsivity control disorder in the network situation, and its essence is the behavioral problem: that the individual lacks the ability to control the network use. ${ }^{62}$ Therefore, self-control can better predict the status of PIU. On this basis, this study also found another pathway for the correlation between self-control and PIU: selfcontrol may indirectly be associated with PIU through rejection sensitivity and loneliness, which is mainly manifested as partial mediating effect of rejection sensitivity, partial mediating effect of loneliness, and a chain mediating effect of rejection sensitivity and loneliness between self-control and PIU. Additionally, the mediating effect size accounted for $9.76 \%, 20.73 \%$, and $4.88 \%$ of the total effect. The relative mediation effect of indirect effect 2 was more than $20 \%$, and indirect effect 1 and indirect effect 3 were relatively small. Therefore, loneliness played the most important mediating role in self-control and PIU.

Although the proportion of indirect effect 1 was small (9.76\%), the results were significant. This finding contains a certain theoretical significance in exploring the relationship between self-control and PIU. People's need to belong is one of the basic psychological needs; generally, people have a strong desire to form and maintain lasting interpersonal relationships. ${ }^{20}$ However, because of the existence of rejection sensitivity, some college students over expect rejection information in interpersonal communication, thereby showing excessive ingratiation, social withdrawal, or other uncomfortable reactions, which leads to further rejection that damages interpersonal relations. ${ }^{73}$ When actual interpersonal needs cannot be satisfied, college students often turn to the Internet to satisfy their communication needs. The theory of compensatory use of the Internet suggests that the risk of negative outcomes (PIU) may be higher when the motivation to go online is based on unsatisfied needs in real life or on certain psychological characteristics. ${ }^{39}$ The cognitive affective processing model theory holds that rejection sensitivity is an interrelated dynamic model in the cognitive affective processing system. Self-control and attention mechanisms may interact with these dynamics as buffers against high rejection sensitivity. Individuals with a high level of self-control are better at using cognitive evaluation strategies, which can suppress irrational cognition and reduce irrational expectations of others' rejection. ${ }^{43}$ This feedback on interpersonal information affects the environment (interpersonal relationship) in the interaction cycle, and makes individuals actively participate in interpersonal interaction, ${ }^{44}$ thus showing lower network dependence. Network behavior is accompanied by great impulsiveness and enjoyment. Individuals' lack of self-control ability makes it difficult for them to supervise and regulate their own cognition, emotion, and behavior in a timely and effective manner. They tend to follow their own impulse, seek immediate satisfaction, and invest too much time and energy into the Internet, which is associated with higher rates of PIU. ${ }^{74}$

According to the analysis of mediating effect, the proportion of indirect effect 2 was $20.73 \%$, which is much higher than the other two paths. Therefore, as a mediator, loneliness plays the most important role in the relationship between self-control and PIU. Loneliness has been associated with negative interpersonal experience for decades. ${ }^{75}$ Loneliness reflects a kind of interpersonal relationship defect, caused by dissatisfaction with a real interpersonal relationship; this further increases loneliness. ${ }^{76}$ Studies have found that Internet addicts are more likely to feel lonely because of poor interpersonal relationships; thus, they escape reality and seek acceptance online. ${ }^{77,78}$ However, the Internet cannot solve the problem of loneliness. ${ }^{79}$ Those who use the Internet as an escape from reality tend to show poorer interpersonal relationships, ${ }^{80}$ deeper loneliness, ${ }^{81}$ and consequently, more PIU. The behavioral cognitive model of PIU believes that social psychological problems (such as loneliness and depression) make some Internet users more susceptible to maladaptive cognition and behavior. ${ }^{30}$ Lonely individuals believe "Nobody loves me offline" and "The Internet is my only friend." Such false cognitive beliefs are considered false cognitive disorder that reinforces the individual's Internet dependence. ${ }^{30}$ Caplan explained the problem of PIU from the perspective of social skills, hypothesizing that individuals with deep loneliness will be drawn toward Internet social networking because they are aware of their lack of social skills; consequently, they tend to use the Internet for social interaction, rather than face-to-face contact, thereby leading to PIU. ${ }^{48}$ All theories and studies mentioned above confirm the relationship between loneliness and PIU in this study. Selfcontrol plays a role in loneliness. According to a developmental cascade model of self-control and loneliness, over time, self-control will affect the level of loneliness through prosocial behavior and peer preference. ${ }^{82}$ This, in turn, is directly related to PIU. The Internet Satisfaction Theory notes that people use the Internet to satisfy different levels of needs; maintaining interpersonal relationships is the most prominent psychological need. ${ }^{83}$ When individuals are dissatisfied 
with interpersonal relationships, those with low self-control levels may experience more negative emotions, such as loneliness. To escape painful experiences of real-world situations, the virtual world then becomes the outlet, causing individuals to excessively use the Internet without restraint. ${ }^{84}$ However, those with high self-control have stronger social skills and can perceive better when their behaviors are not socially acceptable. ${ }^{85}$ They consciously manage their selfimage and invest more cognitive and emotional resources in social interactions. They use social skills effectively and have better experiences of social interaction. ${ }^{86}$ Some studies have found that better social interaction is associated with reduced loneliness. ${ }^{87}$ Additionally, people with good social skills can avoid loneliness, even if they have high social needs, which may keep them from resorting to the Internet, and can partake in real-world social interactions. ${ }^{88}$ Therefore, the occurrence of PIU can be reduced. In addition, the power model of self-control states that inadequate self-regulation occurs when a person has no ability to rise above negative perceptions associated with feeling lonely, leading to exhaustion of self-regulation power resources. The interaction between cognitive failure and depletion of selfregulatory powers results in an individual not having the cognitive powers to overcome the impulses they wish to control (eg, PIU). ${ }^{89}$ The above conclusions support the result of effect 2 in this study, that loneliness plays an important role in self-control and PIU.

The social cognition model of rejection sensitivity believes that early experiences of rejection affect the cognition, emotion, and behavior of individuals; this causes them to have an anxious expectation that others will reject them in communication situations. ${ }^{57}$ They are more passive in interpersonal communication, often cannot obtain satisfactory social contact, and have more negative emotional experiences, such as loneliness. To cope with this negative emotional experience, individuals often turn to the online world ${ }^{90}$ because it creates a social environment where they can communicate with others. ${ }^{91}$ However, they tend to overuse and over rely on the Internet, which is associated with high rates of PIU. ${ }^{92}$ Self-control can adjust cognition and emotion through cognitive evaluation strategy. ${ }^{43}$ With a high level of self-control, individuals can suppress the cognition and emotions caused by rejection, rationally treat interpersonal communication, and experience less loneliness, thereby reducing their dependence on the Internet. When individuals with low self-control experience rejection similar to what they have experienced in the past, the original painful experience is re-lived and the individual experiences dissatisfaction with their interpersonal relationship. This dissatisfaction makes them feel lonelier, and then they rely on the Internet to make up for the real unfulfilled relationship needs.

Considering the complexity of the influence of self-control on PIU, this study creatively used self-control as an independent variable to explore its influence on PIU. This is an innovation in theoretical research related to the influence of self-control on PIU, which not only enriches the relevant research theories on the latter, but also provides a direction for follow-up research. Concurrently, this study also provides a reference point for prevention and offers the following possibilities for interventions for PIU in college students. This can be done as follows. First, cultivate college students' self-control, strengthen their Internet security knowledge, improve their understanding of PIU, and consciously control the frequency of Internet use. Second, create a good interpersonal environment for students. For those with weak selfcontrol, a positive interpersonal environment can help them overcome their escapism stemming from their fear of rejection. This will reduce the likelihood of PIU. Finally, conduct rich cultural activities on campus. Thus when college students with weak self-control encounter setbacks in interpersonal communication, they will have an alternative outlet through which to regulate their emotions and will not fall into loneliness, which can reduce their risk of PIU.

\section{Limitations and Recommendations}

First, the relationship between self-control and PIU is complex and diverse with many mediating variables. This study explored only the role of rejection sensitivity and loneliness. Future research is needed to explore a deeper and more comprehensive relationship between self-control and PIU. Concurrently, when discussing the mediating effect of loneliness, this study defaults to the concept of loneliness as the negative emotion generated by dissatisfaction with interpersonal relationships and considers the mediating effect of loneliness from a one-dimensional perspective. However, loneliness is a complex structure related to the environment and different factors, such as parents and peers. ${ }^{93}$ The identification of specific determinants (peers or parents) of problematic Internet use is of great significance for prevention and intervention. ${ }^{4}$ Therefore, future studies should consider the complex structure of loneliness to explore its influence on PIU. Second, it is difficult to reveal the causal relationship between self-control and PIU when adopting a cross-sectional study. Future research is expected 
to use longitudinal research, experimental research, and other methods to reveal the method of self-control more accurately on PIU. Finally, this study is an empirical study based on data investigation. In the future, more intervention studies are expected to help in the reduction of PIU among college students by improving self-control, reducing rejection sensitivity, and reducing loneliness.

\section{Conclusion}

Self-control can affect PIU in college students, through three indirect paths,: the mediating role of rejection sensitivity and loneliness, the chain mediating roles of rejection sensitivity and loneliness, and the mediating effect size, accounting for $9.76 \%, 20.73 \%$, and $4.88 \%$ of the total effect, respectively. Indirect effect 2 (self-control $\rightarrow$ loneliness $\rightarrow$ PIU) accounted for the largest proportion of the total effect. Therefore, loneliness plays the most important role in the relationship between self-control and PIU. The other two indirect effects accounted for a small proportion of the total effect, and the results were significant. These results suggest that self-control can affect PIU through rejection sensitivity and other factors as well.

\section{Ethics Statement}

Our research was carried out through the Internet. The online questionnaire is anonymous and the information of all participants is protected. In the first part of the online questionnaire, participants were fully informed of the survey before participation, such as content and purpose. Completing and submitting the questionnaire was regarded as their proxy consent to participate. All participants were informed and agreed to the above. This study was conducted in accordance with the Declaration of Helsinki. The Ethics Committee of College of Teacher Education at Ningbo University approved the study.

\section{Author Contributions}

All authors made a significant contribution to the work reported, whether that is in the conception, study design, execution, acquisition of data, analysis and interpretation, or in all these areas; took part in drafting, revising or critically reviewing the article; gave final approval of the version to be published; have agreed on the journal to which the article has been submitted; and agree to be accountable for all aspects of the work.

\section{Funding}

This study was supported by the Education Department of Zhejiang Province (grant number Y201840696).

\section{Disclosure}

The authors declare that the research was conducted in the absence of any commercial or financial relationships that could be construed as a potential conflict of interest.

\section{References}

1. Musetti A, Corsano P. The Internet is not a tool: reappraising the model for Internet-addiction disorder based on the constraints and opportunities of the digital environment. Front Psychol. 2018;9:558. doi:10.3389/fpsyg.2018.00558

2. Zhun G, Liyun W, Haijiao W. Perceived stress and PIU among Chinese college students: mediating effect of procrastination and moderating effect of flow. Front Psychol. 2021;12. doi:10.3389/fpsyg.2021.632461

3. China Internet Network Information Center. The 48th statistical report on Internet development in China, 2021-09-15; 2021. Available from: http:// www.cnnic.net.cn/hlwfzyj/hlwxzbg/hlwtjbg/202109/t20210915_71543.htm.pdf. Accessed September 15, 2021.

4. Musetti A, Corsano P, Boursier V, Schimmenti A. Problematic Internet use in lonely adolescents: the mediating role of detachment from parents. Clin Neuropsychiatry. 2020;17:3-10. doi:10.36131/clinicalnpsych20200101

5. Niu G, Sun X, Zhou Z, Wei H. A review of cognitive neuroscience studies on Internet addiction. Adv Psychol Sci. 2013;21:1104-1111. doi:10.3724/ sp.j.1042.2013.01104

6. Zhang J, Chen C, Wang L, et al. The relationship between time spent online and Internet addiction among Chinese college freshmen: a mediated moderation model. Acta Psychol. 2014;46:1521-1533. doi:10.3724/SP.J.1041.2014.0152

7. Block JJ. Issues for DSM-V: internet addiction. Am J Psychiatry. 2008;165:306-307. doi:10.1176/appi.ajp.2007.07101556

8. Schimmenti A, Passanisi A, Gervasi AM, Manzella S, Famà FI. Insecure attachment attitudes in the onset of problematic Internet use among late adolescents. Child Psychiatry Hum Dev. 2014;45:588-595. doi:10.1007/s10578-013-0428-0

9. Young KS. Tangled in the Web: Understanding Cybersex from Fantasy to Addiction. Boomington: IN: Authorhouse; 2001. 
10. Musetti A, Cattivelli R, Giacobbi M, et al. Challenges in Internet addiction disorder: is a diagnosis feasible or not?. Front Psychol. $2016 ; 7: 842$. doi:10.3389/fpsyg.2016.00842

11. Goto Y. The negative influence of Internet addiction on morality of Japanese youth: loss of relationships with others and oneself. Habitus. 2017;21:73-87. doi:10.15027/42918

12. Kormas G, Critselis E, Janikian M, Kafetzis D, Tsitsika A. Risk factors and psychosocial characteristics of potential problematic and problematic Internet use among adolescents: a cross-sectional study. BMC Public Health. 2011;11. doi:10.1186/1471-2458-11-595

13. Kandell JJ. Internet addiction on campus: the vulnerability of college students. Cyber Psychol Behav. 1998;1:11-17. doi:10.1089/cpb.1998.1.11

14. Milani L, La Torre G, Fiore M, et al. Internet gaming addiction in adolescence: risk factors and maladjustment correlates. Int $J$ Ment Health Addict. 2017;16:888-904. doi:10.1007/s11469-017-9750-2

15. Saikia A, Das J, Barman P, Bharali M. Internet addiction and its relationships with depression, anxiety, and stress in urban adolescents of Kamrup District, Assam. J Fam Commun Med. 2019;26:108-112. doi:10.4103/jfcm.JFCM_93_18

16. Sun Y, Wang X, Liu S, Li Z. The brain mechanism on working memory level of Internet addicts: from the evidence of ERP. $J$ Psychol Sci. 2017;40:1208-1214. doi:10.3724/SP.J.1041.2020.013

17. Jia Y, An L, Jia Y. Social competence and Internet addiction: chain mediation of social adaptation and inferiority. Chin J Clin Psychol. 2019;27:103-107. doi:10.16128/j.cnki.1005-3611.2019.01.021

18. Chen Y, Li R, Zhang P, Liu X. Headmaster's autonomy support, involvement, warmth and adolescents' Internet addiction: a moderated mediating model. Chin J Clin Psychol. 2019;27:306-310. doi:10.16128/j.cnki.1005-3611.2019.02.020

19. Van Damme S, Crombez G, Goubert L, Eccleston C. Current issues and new directions in psychology and health: the costs and benefits of selfregulation — a call for experimental research. Psychol Health. 2009;24:367-371. doi:10.1080/08870440902866902

20. Baumeister RF, Leary MR. The need to belong: desire for interpersonal attachments as a fundamental human motivation. Psychol Bull. 1995;117:497-529. doi:10.1037/0033-2909.117.3.497

21. Kim EJ, Namkoong K, Ku T, Kim SJ. The relationship between online game addiction and aggression, self-control and narcissistic personality traits. Eur Psychiatry. 2008;23:212-218. doi:10.1016/j.eurpsy.2007.10.010

22. He C, Xia M, Jiang G, Wei H. Mediation role of self-control between Internet game addiction and self-esteem. Chin J Clin Psychol. 2012;20:58-60. doi:10.16128/j.cnki.1005-3611.2012.01.011

23. Özdemir Y, Kuzucu Y, Ak Ş. Depression, loneliness and Internet addiction: how important is low self-control?. Comput Hum Behav. 2014;34:284-290. doi:10.1016/j.chb.2014.02.009

24. Hofmann W, Friese M, Strack F. Impulse and self-control from a dual-systems perspective. Perspect Psychol Sci. 2009;4:162-176. doi:10.2307/ 40212310

25. Baumeister RF, DeWall CN, Ciarocco NJ, Twenge JM. Social exclusion impairs self-regulation. J Pers Soc Psychol. $2005 ; 88: 589-604$. doi:10.1037/0022-3514.88.4.589

26. Baumeister RF, Vohs KD, Tice DM. The strength model of self-control. Curr Direct Psychol Sci. 2007;16:351. doi:10.1111/j.14678721.2007.00534.x

27. Xiang M, Zhang L. The strength model of self-control: progress in the field of sport. China Sport Sci. 2016;8:67-78. doi:10.16469/j.css.201608006

28. Baumeister RF, Gailliot M, DeWall CN, Oaten M. Self-regulation and personality: how interventions increase regulatory success, and how depletion moderates the effects of traits on behavior. J Pers. 2006;74:1773-1802. doi:10.1111/j.1467-6494.2006.00428.x

29. Cole SH, Hooley JM. Clinical and personality correlates of MMO gaming: anxiety and absorption in problematic Internet use. Soc Sci Comput Rev. 2013;31:424-436. doi:10.1177/0894439312475280

30. Davis RA. A cognitive-behavioral model of pathological Internet use. Comput Human Behav. 2001;17:187-195. doi:10.1016/s0747-5632(00) 00041-8

31. Downey G, Feldman SI. Implications of rejection sensitivity for intimate relationships. J Pers Soc Psychol. 1996;70:1327-1343. doi:10.1037/00223514.70.6.1327

32. Ayduk O, Downey G, Kim M. Rejection sensitivity and depressive symptoms in women. Pers Soc Psychol Bull. 2001;27:868-877. doi:10.1177/ 0146167201277009

33. Watson J, Nesdale D. Rejection sensitivity, social withdrawal, and loneliness in young adults. J Appl Soc Psychol. 2012;42:1984-2005. doi:10.1111/j.1559-1816.2012.00927.x

34. London B, Downey G, Bonica C, Paltin I. Social causes and consequences of rejection sensitivity. J Res Adolsc. 2007;17:481-506. doi:10.1111/ j.1532-7795.2007.00531.x

35. Ko CH, Liu TL, Wang PW, Chen CS, Yen CF, Yen JY. The exacerbation of depression, hostility, and social anxiety in the course of Internet addiction among adolescents: a prospective study. Comprehens Psychiatry. 2014;55:1377-1384. doi:10.1016/j.comppsych.2014.05.003

36. Molavi P, Mikaeili N, Ghaseminejad MA, Kazemi Z, Pourdonya M. Social anxiety and benign and toxic online self-disclosures: an investigation into the role of rejection sensitivity, self-regulation, and Internet addiction in college students. J Nerv Ment Dis. 2018;206:598-605. doi:10.1097/ NMD.0000000000000855

37. Sun X, Wu M. Relationships between self-esteem, rejection sensitivity, interpersonal trust and social anxiety of undergraduates. Chin $J$ Clin Psychol. 2011;19:537-539. doi:10.16128/j.cnki.1005-3611.2011.04.012

38. Tan S, Guo Y. Revision of self-control scale for Chinese college students. Chin J Clin Psychol. 2008;16:468-470. doi:10.16128/j.cnki.10053611.2008.05.022

39. Kardefelt-Winther D. Problematizing excessive online gaming and its psychological predictors. Comput Hum Behav. 2014;31:118-122. doi:10.1016/j.chb.2013.10.017

40. Knowles ML, Haycock N, Shaikh I. Does Facebook magnify or mitigate threats to belonging? Soc Psychol. 2015;46:313-324. doi:10.1027/18649335/a000246

41. Bland ED. An appraisal of psychological \& religious perspectives of self-control. J Relig Health. 2008;47:416. doi:10.2307/40344418

42. Ayduk ON. Impact of self-control strategies on the link between rejection sensitivity and hostility: risk negotiation through strategic control. Columbia University. ProQuest Dissertations Publishing; 1999:9916856.

43. Ayduk O, Mendoza-Denton R, Mischel W, Downey G, Peake PK, Rodriguez M. Regulating the interpersonal self: strategic self-regulation for coping with rejection sensitivity. J Pers Soc Psychol. 2000;79:776-792. doi:10.1037/0022-3514.79.5.776 
44. Ayduk Ö, Gyurak A. Applying the cognitive-affective processing systems approach to conceptualizing rejection sensitivity. Soc Pers Psychol Comp. 2008;2:2016-2033. doi:10.1111/j.1751-9004.2008.00143.x

45. Weiner MF. Loneliness: the experience of emotional and social isolation. Int J Group Psychother. 1975;25:239-240. doi:10.2307/2062224

46. Zhao L, Chen S, Su W, Ren Z. A meta-analysis of the relationship between loneliness and Internet addiction and its related factors. Chin J Clin Psychol. 2016;30:554-560. doi:10.3969/j.issn.1000-6729.2016.07.014

47. Caplan SE. Preference for online social interaction: a theory of problematic internet use and psychosocial well-being. Communic Res. 2003;30 (6):625-648. doi:10.1177/0093650203257842

48. Caplan SE. A social skill account of problematic internet use. J Communic. 2005;55(4):721-736. doi:10.1111/j.1460-2466.2005.tb03019.x

49. Davis RA, Flett GL, Besser A. Validation of a new scale for measuring problematic internet use: implications for pre-employment screening. CyberPsychol Behav. 2002;5(4):331-345. doi:10.1089/109493102760275581

50. Zhen Z, Chen M, Wang M, Yan J. Relationships among rejection sensitivity and pathological Internet use: chain mediating effect of self-esteem and loneliness. Chin J Health Psychol. 2019;27:1890-1894. doi:10.13342/j.cnki.cjhp.2019.12.035

51. Ang CS, Chan NN, Lee CS. Shyness, loneliness avoidance, and Internet addiction: what are the relationships? J Psychol. 2018;152:25-35. doi:10.1080/00223980.2017.1399854

52. Peper E, Harvey R. Digital addiction increases loneliness, anxiety and depression. NewsRx Health Sci. 2018;5:3-8. doi:10.15540/nr.5.1.3

53. Kocher MG, Martinsson P, Myrseth KOR, Wollbrant CE. Strong, bold, and kind: self-control and cooperation in social dilemmas. Exp Econ. 2017;20(1):44-69. doi:10.1007/s10683-015-9475-7

54. Denson TF, DeWall CN, Finkel EJ. Self-control and aggression. Curr Dir Psychol Sci. 2012;21(1):20-25. doi:10.1177/0963721411429451

55. Stavrova O, Ren D, Pronk T. Low self-control: a hidden cause of loneliness? Pers Soc Psychol Bull. 2021;014616722110072. doi:10.1177/ 01461672211007228

56. Bahr HM, Peplau LA, Perlman D. Loneliness: a sourcebook of current theory, research and therapy. Contemp Sociol. 1984;13:203. doi:10.2307/ 2068915

57. Romero-Canyas R, Downey G, Berenson K, Ayduk O, Kang NJ. Rejection sensitivity and the rejection-hostility link in romantic relationships. J Pers. 2010;78:119-148. doi:10.1111/j.1467-6494.2009.00611.x

58. Schoemann AM, Boulton AJ, Short SD. Determining power and sample size for simple and complex mediation models. Soc Psychol Personal Sci. 2017:194855061771506. doi:10.1177/1948550617715068

59. Tangney JP, Baumeister RF, Boone AL. High self-control predicts good adjustment, less pathology, better grades, and interpersonal success. J Pers. 2004;72:271-324. doi:10.1111/j.0022-3506.2004.00263.x

60. Russell D, Peplau LA, Cutrona CE. The revised UCLA Loneliness Scale: concurrent and discriminant validity evidence. J Pers Soc Psychol. 1980;39:472-480. doi:10.1037/0022-3514.39.3.472

61. Wang D. Study on the reliability and validity of Russell Loneliness Scale. Chin J Clin Psychol. 1995;1:23-25. doi:10.16128/j.cnki.10053611.1995.01.006

62. Young KS. Internet addiction: the emergence of a new clinical disorder. CyberPsychol Behav. 1998;1:237-244. doi:10.3329/akmmcj.v10i2.44116

63. Mei S, Chai J, Guo J. Subjective well-being and Internet addiction in adolescents: mediating effects of self-esteem and self-control. Psychol Dev Educ. 2015;31:603-609. doi:10.16187/j.cnki.issn1001-4918.2015.05.12

64. Zhou H, Long L. Statistical remedies for common method biases. Adv Psychol Sci. 2004;12:942-950.

65. Gu H, Wen Z. Reporting and interpreting multidimensional test scores: a bi-factor perspective. Psychol Dev Educ. 2017;33:504-512. doi:10.16187/ j.cnki.issn1001-4918

66. Wen ZL, Hau KT, Chang L. A comparison of moderator and mediator and their applications. Acta Psychol Sinica. 2005;37:268-274.

67. Lau RS, Cheung GW. Estimating and comparing specific mediation effects in complex latent variable models. Organ Res Meth. 2012;15(1):3-16. doi:10.1177/1094428110391673

68. Preacher KJ, Hayes AF. Asymptotic and resampling strategies for assessing and comparing indirect effects in multiple mediator models. Behav Res Meth. 2008;40:879-891. doi:10.3758/brm.40.3.879

69. Wen Z, Ye B. Analyses of mediating effects: the development of methods and models. Adv Psychol Sci. 2014;22:731-745. doi:10.3724/SP. J.1042.2014.00731

70. Li H, Guo Y, Yu Q. Self-control makes the difference: the psychological mechanism of dual processing model on Internet addicts' unusual behavior in intertemporal choice. Comput Hum Behav. 2019;101:95-103. doi:10.1016/j.chb.2019.07.010

71. Song WJ, Park JW. The influence of stress on Internet addiction: mediating effects of self-control and mindfulness. Int J Ment Health Addict. 2019;17:1557-1874. doi:10.1007/s11469-019-0051-9

72. Gottfredson MR, Hirschi T. A General Theory of Crime. Stanford: Stanford University Press; 1990.

73. Romero-Canyas R, Downey G, Reddy KS, Rodriguez S, Cavanaugh TJ, Pelayo R. Paying to belong: when does rejection trigger ingratiation? J Pers Soc Psychol. 2010;99:802-823. doi:10.1037/a0020013

74. Nie Y, Dou K, Wang Y. Impulsive and Internet addition disorder: a mediator role of self-control. J Ningbo Univ. 2013;35:7-12. doi:10.16719/j. cnki.1671-6981.20190526

75. Malerstein AJ. Loneliness: the experience of emotional and social isolation. Am J Psychother. 1976;30:685-686. doi:10.1080/ 00207284.1975 .11491894

76. Ponzetti JJ. Loneliness among College Students. Fam Relat. 1990;39(3):336-340. doi:10.2307/584881

77. Wang H, Zhou X, Lu C, et al. Problematic internet use in high school students in Guangdong Province, China. PLoS One. 2011;6(5):e19660. doi:10.1371/journal.pone.0019660

78. Hou J, Jiang Y, Chen S, et al. Cognitive mechanism of intimate interpersonal relationships and loneliness in internet-addicts: an ERP study. Addict Behav Rep. 2019;10:100209. doi:10.1016/j.abrep.2019.100209

79. Leung L. Net-generation attributes and seductive properties of the internet as predictors of online activities and internet addiction. CyberPsychol Behav. 2004;7(3):333-348. doi:10.1089/1094931041291303

80. Milani L, Osualdella D, Di Blasio P. Quality of interpersonal relationships and problematic internet use in adolescence. CyberPsychol Behav. 2009;12(6):681-684. doi:10.1089/cpb.2009.0071 
81. Ceyhan AA, Ceyhan E. Loneliness, depression, and computer self-effificacy as predictors of problematic Internet use. Cyberpsycho Behav. 2008;11:699-701. doi:10.1089/cpb.2007.0255

82. Liu J, Xiao B, Hipson WE, Coplan RJ, Li D, Chen X. Self-control, peer preference, and loneliness in Chinese children: a three-year longitudinal study. Social Development. 2016. doi:10.1111/sode.12224

83. Ruggiero TE. Uses and gratifications theory in the 21st century. Mass Commun Soc. 2000;3:3-37. doi:10.1207/S15327825MCS0301_02

84. Ryu H, Kim H. The relationship between self-control and Internet game addiction proneness in college students: dual mediating effects of perceived stress and coping strategy. Korean J Clin Psychol. 2018;37:156-165. doi:10.15842/kjcp.2018.37.2.003

85. Smith CV, Lair EC, O’Brien SM. Purposely stoic, accidentally alone? Self-monitoring moderates the relationship between emotion suppression and loneliness. Pers Ind Diff. 2019;149:286-290. doi:10.1016/j.paid.2019.06.012

86. Ickes W, Holloway R, Stinson LL, Hoodenpyle TG. Self-monitoring in social interaction: the centrality of self-affect. J Pers. 2006 ;74:659-684. doi:10.1111/j.1467-6494.2006.00388.x

87. Gable SL, Reis HT. Good news! Capitalizing on positive events in an interpersonal context. Adv Exp Soc Psychol. 2010;42:195-257. doi:10.1016/ S0065-2601(10)42004-3

88. Prievara DK, Piko BF, Luszczynska A. Problematic Internet use, social needs, and social support among youth. Int J Ment Health Addict. 2019;17:1008-1019. doi:10.1007/s11469-018-9973-x

89. McQuade A, Gill P. The role of loneliness and self-control in predicting problem gambling behaviour. Gambl Res. 2012;24(1):18-30. doi:10.3316/ informit.859155913483693

90. Muñoz-Rivas MJ, Fernández L, Gámez-Guadix M. Analysis of the indicators of pathological Internet use in Spanish university students. Span J Psychol. 2010;13:697-707. doi:10.1017/S1138741600002365

91. Morahan-Martin J, Schumacher P. Incidence and correlates of pathological Internet use among college students. Comput Hum Behav. 2000;16:13-29. doi:10.1016/s0747-5632(99)00049-7

92. Erol O, Cirak NS. Exploring the loneliness and Internet addiction level of college students based on demographic variables. Contemp Educ Technol. 2019;10:156-172. doi:10.30935/cet.554488

93. Goossens L, Beyers W. Comparing measures of childhood loneliness: internal consistency and confirmatory factor analysis. J Clin Child Adol Psychol. 2002;31:252-262. doi:10.1207/S15374424JCCP3102_10

\section{Publish your work in this journal}

Psychology Research and Behavior Management is an international, peer-reviewed, open access journal focusing on the science of psychology and its application in behavior management to develop improved outcomes in the clinical, educational, sports and business arenas. Specific topics covered in the journal include: Neuroscience, memory and decision making; Behavior modification and management; Clinical applications; Business and sports performance management; Social and developmental studies; Animal studies. The manuscript management system is completely online and includes a very quick and fair peer-review system, which is all easy to use. Visit http://www.dovepress.com/testimonials.php to read real quotes from published authors.

Submit your manuscript here: https://www.dovepress.com/psychology-research-and-behavior-management-journal 